\title{
Age-associated difference in circulating ACE2, the gateway for SARS-COV-2, in humans: results from the InCHIANTI study
}

\author{
Majd AlGhatrif $(\mathbb{D} \cdot$ Toshiko Tanaka Ann Zenobia \\ Moore • Stefania Bandinelli • Edward G. Lakatta • \\ Luigi Ferrucci
}

Received: 18 August 2020 / Accepted: 9 December 2020 / Published online: 18 January 2021

(C) This is a U.S. government work and not under copyright protection in the U.S.; foreign copyright protection may apply 2021

\begin{abstract}
Levels of angiotensin-converting enzyme 2 (ACE2), the gateway for COVID-19 virus into the cells, have been implicated in worse COVID-19 outcomes associated with aging and cardiovascular disease (CVD). Data on age-associated differences in circulating ACE2 levels in humans and the role of CVD and medications is limited. We analyzed data from 967 participants of the InCHIANTI study, a communitydwelling cohort in the Chianti region, Italy. Relative abundance of ACE2 in plasma was assessed using a proteomics platform. CVD diagnoses, use of reninangiotensin-aldosterone system (RAAS) antagonists: ACEi, ARBs, and aldosterone antagonists, were ascertained. Multiple linear analyses were performed to examine the independent association of ACE2 with age, CVD, and RAAS antagonist use. Age was independently associated with lower log (ACE2) in persons
\end{abstract}

M. AlGhatrif $(\bowtie) \cdot$ E. G. Lakatta $\cdot$ L. Ferrucci

Laboratory of Cardiovascular Science, National Institute on

Aging, National Institutes of Health, 251 Bayview Blvd.,

Baltimore, MD 21224, USA

e-mail: Majd.alghatrif@nih.gov

M. AlGhatrif · T. Tanaka · A. Z. Moore

Longitudinal Study Section, National Institute on Aging, National Institutes of Health, Baltimore, MD, USA

M. AlGhatrif

Divisions of Cardiology and Hospital Medicine, Department of Medicine, Johns Hopkins School of Medicine, Baltimore, MD, USA

S. Bandinelli

Geriatric Unit, ASL Toscana Centro, Florence, Italy aged $\geq 55$ years (STD $\beta=-0.12, p=0.0002)$. ACEi treatment was also independently associated with significantly lower ACE2 levels, and ACE2 was inversely associated with weight, and positively associated with peripheral artery disease (PAD) status. There was a trend toward higher circulating ACE2 levels in hypertensive individuals, but it did not reach statistical significance. In a stratified analysis, the association between $\log$ (ACE2) and $\log$ (IL-6) was more evidenced in participants with PAD. Circulating ACE2 levels demonstrate curvilinear association with age, with older individuals beyond the sixth decade age having lower levels. ACEi was associated with greater circulating ACE2 levels. Interestingly, ACE2 was elevated in PAD and positively associated with inflammatory markers, suggesting compensatory upregulation in the setting of chronic inflammation. Further studies are needed to comprehensively characterize RAAS components with aging and disease, and assess its prognostic role in predicting COVID-19 outcomes.

Keywords COVID-19 · Aging · Cardiovascular disease $\cdot$ ACE2

\section{Introduction}

With the surging death toll of the COVID-19 pandemic around the world, older people, especially those with cardiovascular diseases, have emerged as the group at highest risk for severe COVID-19 illness and mortality when compared to the general population $[1,2]$. On the 
other hand, the growing community COVID-19 testing data revealed a curvilinear relationship between age and disease incidence, with the lowest rates being among children, followed by an increase in young adults, to decline after the sixth decade, with another peak in the 8th decade of age [3]. While multiple social and environmental factors can contribute to this age distribution, the age difference of COVID-19 incidence compared to other seasonal respiratory diseases [4], and the paradoxical association of age with disease incidence and severity, warrants investigating other biological factors [5]. The angiotensin-converting enzyme 2 (ACE2) that serves as a gateway for the virus to enter the cells [6] is a key anti-inflammatory component of the reninangiotensin-aldosterone system (RAAS). Thus, ACE2 potentially may be a determinant of age-associated predisposition for COVD-19 incidence and severity [5]. However, data examining ACE2 changes with aging are still limited.

An earlier animal study examining age change in ACE2 [7], in the context of the 2003 SARS-COV, which had higher incidence in younger adults as well [8], has shown greater rates of ACE2 expression in the lungs of younger vs. older rats [7]. A more recent study of a predominantly young cohort has shown that children $<10$ years, the age group with the lowest ACE2 incidence, have lower ACE2 gene expression in nasal epithelium compared to their older counterparts [9]. In the former study, ACE2 nasal gene expression increased linearly from $<10$ to $18-24$ years of with less pronounced increase among those aged 25 years or older. Another study of 23 healthy human subjects reported an inverse association between ACE2 and ACE ratio levels, i.e. a relative decline in ACE2, and aging in hematopoietic stem progenitor cells [10].

Given the importance of this topic and scarcity of information about it, large-scale studies examining ageassociated difference in ACE2 are urgently needed. In this analysis, we examined age-associated differences in circulating ACE2 levels in a community-dwelling population, exploring the effects of other factors affecting ACE2 levels such as RAAS antagonists and cardiovascular risk factors and diseases [11]. In this analysis, we aimed to examine age-associated differences in circulating ACE2 level in a community-dwelling population and examine the association between cardiovascular morbidities and RAAS antagonists with circulating ACE2 levels in this population.

\section{Methods}

Sample

We analyzed data from 967 participants of the "Invecchiare in Chianti" (Aging in the Chianti Area, InCHIANTI) study. The InCHIANTI study is a population-based epidemiological study aimed at evaluating factors that influence mobility in the older population living in the Chianti region in Tuscany, Italy. Details of the study have been previously reported [12]. Briefly, 1616 residents were selected from the population registry of Greve in Chianti (a rural area: 11,709 residents with $19.3 \%$ of the population older than 65 years of age) and Bagno a Ripoli (Antella village near Florence; 4704 inhabitants, with 20.3\% older than 65 years of age). The participation rate was $90 \%(n=1453)$ and subjects' age ranged between 21 and 102 years. The study protocol was approved by the Italian National Institute of Research and Care of Aging Institutional Review and Medstar Research Institute (Baltimore, MD) and approved by the Internal Review Board of the National Institute for Environmental Health Sciences (NIEHS). All participants provided written informed consent.

\section{Assessment of laboratory measures}

Circulating ACE2 abundances were assessed using the 1.3k SOMAscan Assay at the Trans-NIH Center for Human Immunology, Autoimmunity, and Inflammation (CHI), National Institute of Allergy and Infectious Diseases, National Institutes of Health (Bethesda, MD, USA) using the same methods and data normalization process as previously [13]. From the $1.3 \mathrm{k}$ panel, the probe for ACE2 (SL004415) was used for subsequent analyses. Specifically, relative abundance of circulating ACE2 in plasma was assessed using aptamer-based proteomics platform and values were log transformed. Inflammatory markers were measured in blood samples drawn in the morning after a 12-h overnight fast and resting period. Aliquots of serum were stored at $-80^{\circ} \mathrm{C}$. Serum interleukin-6 (IL-6) was measured in duplicate by high-sensitivity enzyme-linked immunosorbent assays (ELISAs; kits from BIOSOURCE, Camarillo, CA) with a sensitivity of $0.1 \mathrm{pg} / \mathrm{mL}$ and an intra-assay coefficient of variations less than $6 \%$. CRP was measured using a high-sensitivity ELISA, a competitive immunoassay that uses purified protein and polyclonal anti-CRP 
antibodies (sensitivity $0.03 \mu \mathrm{g} / \mathrm{mL}$ and inter-assay coefficient of variations $<5 \%$ ). Serum creatinine was measured by commercial enzymatic assay using a Modular P800 Hitachi analyzer. Creatinine clearance was calculated based on 24-h urine collection. Fasting serum glucose level was determined using an enzymatic colorimetric assay (Roche Diagnostics, Mannheim, Germany) and a Roche-Hitachi 917 analyzer.

Medical conditions and medication use

Sociodemographic information (age, sex) was obtained during a structured interview. Clinical diagnoses of CVD of hypertension (HTN), diabetes (DM), ischemic heart disease (IHD), congestive heart failure (CHF), and peripheral arterial disease (PAD defined as ankle-brachial index < 0.9) [14], and the use of RAAS drugs i.e. ACEi, ARBs, and aldosterone antagonists, were ascertained using self-reported medical history, a physical examination, clinical testing including a 12 leads EKG and a measure of the ankle-brachial index, medical records, and laboratory results as described previously [15].

Statistical analysis

A Loess curve was fitted to explore the relationship between log-transformed ACE2 and age, showing a curvilinear relationship with an inflection point in the association around age 55 (Fig. 1); this inflection point is concordant with middle of the age range studied
(55 years, range 20-90). Multiple linear regression model predicting ACE2 with age and age [2] as covariate was fitted to test the significance of the curvilinear relationship observed. The correlations between age and ACE2 were tested using stratified, Spearman's rank-order correlation analysis using age 55 as a threshold to define the age groups. Sensitivity analyses demonstrated consistent results with different thresholds for stratification (50, 55, 60 years); hence, age value of 55 was adopted throughout the analysis. Descriptive statistics as well as $t$ tests and $\chi^{2}$ tests were used to describe the study sample and age strata. Given the small sample size of the younger group, and the low prevalence of the chronic conditions of interests (HTN, DM, and PAD), subsequence multiple linear regression models predicting log (ACE2) were fitted in the older group only; categorical covariates with relative group size ratio greater than $(1: 10)$, i.e. prevalence $<9 \%$, were excluded from the models due to the unreliability of estimates derived from small sample sizes. Covariates were added to subsequent model, and then a final reduced model was generated using backward elimination with a criterion of alpha of 0.1 to enter and remain in the model. The associations between ACE2 and inflammatory markers, IL-6 and CRP, were explored in subsequent multiple linear regression models that incorporated covariates from prior analyses: A final model for IL-6 was evaluated in strata defined by peripheral arterial disease status. ACE2, CRP, and IL-6 were log transformed in all regression analyses.
Fig. 1 Scatterplot with Spearman's rank-order correlations of age vs. ACE2 circulating levels in the different age groups

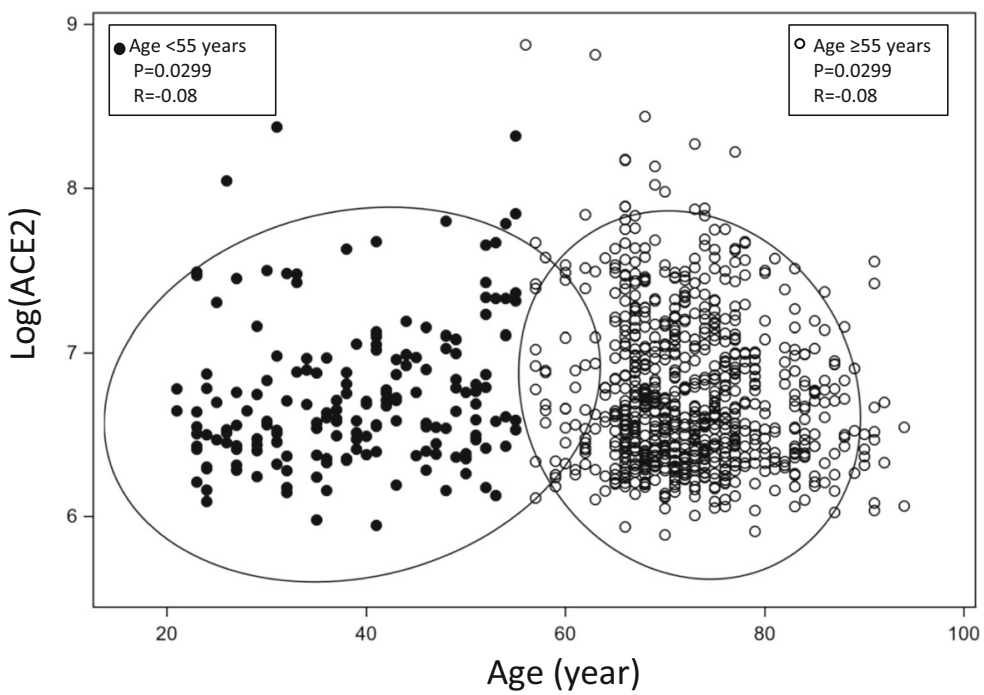




\section{Results}

Sample characteristics of the total sample, and by age groups, are shown in Table 1. As expected, the two age groups differed by average blood pressure, metabolic parameters, smoking, and prevalence of cardiovascular medication use as well as ACEi and ARB drug treatment. Average age groups in log (ACE2) levels were not significantly different (Table 1 ).

To further explore the association between age and ACES, an exploratory Loess curve was fitted, showing a curvilinear relationship with an inflection point in the association around age 55 (Fig. 1). Linear regression analysis of age vs. ACE2 in the total sample, with age entered as a quadratic term, showed significant associations between ACE2 and age $(\beta=0.019, p=0.0013)$, and age [2] $(\beta=0.0002, p=0.0006)$. Stratified,
Spearman rank-order correlation showed a significant positive correlation in the younger group $(R=0.19, p=$ $0.0106)$, and a negative correlation in the older group $(R=-0.08, p=0.0299)$ (Fig. 1).

Subsequent multivariate analyses were limited to the older group of participants aged 55 years and older, given the small size in the younger group, and the low prevalence of categorical variable. A series of hierarchical linear regression models including covariates with prevalence $>9 \%$ were included: Models 1-4 show that the negative association between age and log (ACE2) in this age group remained significant after accounting for covariates, with no sex difference (Table 2). Model 2 shows no independent associations of $\log$ (ACE2) with hypertension and diabetes. Peripheral arterial disease, however, was associated with greater ACE2 levels, while ACEi use was inversely associated with ACE2

Table 1 Descriptive characteristics of the sample by age groups

\begin{tabular}{|c|c|c|c|c|c|c|c|}
\hline \multirow[b]{2}{*}{ Variable } & \multicolumn{2}{|c|}{ Total $(n=967)$} & \multicolumn{2}{|c|}{$<55(n=177)$} & \multicolumn{2}{|c|}{$\geq 55(n=790)$} & \multirow[t]{2}{*}{$p$ value } \\
\hline & Mean $/ n$ & $\mathrm{STD} / \%$ & Mean $/ n$ & $\mathrm{STD} / \%$ & Mean $/ n$ & $\mathrm{STD} / \%$ & \\
\hline AGE, year & 66.3 & 15.2 & 38.6 & 10.0 & 72.5 & 7.0 & $<.0001$ \\
\hline $\mathrm{SBP}, \mathrm{mmHg}$ & 144.2 & 21.2 & 123.6 & 17.6 & 148.8 & 19.2 & $<.0001$ \\
\hline $\mathrm{DBP}, \mathrm{mmHg}$ & 82.6 & 9.3 & 76.3 & 10.3 & 84.0 & 8.4 & $<.0001$ \\
\hline Cr clearance $(\mathrm{mL} / \mathrm{min})$ & 84.9 & 29.3 & 112.7 & 31.9 & 78.9 & 25.0 & $<.0001$ \\
\hline Creatinine & 0.9 & 0.2 & 0.9 & 0.1 & 0.9 & 0.2 & 0.032 \\
\hline Glucose levels & 93.2 & 23.6 & 85.0 & 19.6 & 95.2 & 24.1 & $<.0001$ \\
\hline Total cholesterol & 216.1 & 39.6 & 201.2 & 40.8 & 219.8 & 38.4 & $<.0001$ \\
\hline BMI & 27.2 & 4.1 & 25.8 & 4.1 & 27.5 & 4.1 & $<.0001$ \\
\hline ACE2, RFU & 952.0 & 607.3 & 946.7 & 631.7 & 953.4 & 601.3 & 0.8893 \\
\hline $\operatorname{HTN}(n, \%)$ & 371 & 38.3 & 14 & 7.9 & 357 & 45.2 & 0.0001 \\
\hline $\mathrm{DM}(n, \%)$ & 102 & 10.6 & 4 & 2.3 & 98 & 12.4 & 0.0001 \\
\hline $\operatorname{IHD}(n, \%)$ & 53 & 5.5 & 0 & 0.0 & 53 & 6.7 & 0.0001 \\
\hline $\mathrm{CHF}(n, \%)$ & 28 & 2.9 & 0 & 0.0 & 28 & 3.5 & 0.0001 \\
\hline $\operatorname{PAD}(n, \%)$ & 75 & 7.8 & 0 & 0 & 75 & 9.5 & 0.0001 \\
\hline Non-CVD comorbidities $(n, \%)$ & 49 & 5.1 & 2 & 1.1 & 47 & 6.0 & 0.0082 \\
\hline Smoking $(n, \%)$ & & & & & & & 0.0001 \\
\hline Current & 190 & 19.1 & 70 & 34.3 & 120 & 15.2 & \\
\hline Former & 253 & 25.5 & 31 & 15.2 & 222 & 28.1 & \\
\hline Never & 551 & 55.5 & 103 & 50.5 & 448 & 56.7 & \\
\hline ACEI treatment $(n, \%)$ & 172 & 17.8 & 6 & 3.4 & 166 & 21 & 0.0001 \\
\hline ARB treatment $(n, \%)$ & 12 & 1.24 & 1 & 0.56 & 11 & 0.4 & 0.3688 \\
\hline
\end{tabular}

$N$ number of individuals in the indexed group of categorical variables; \% percentage of individuals in the indexed group of categorical variables; $S T D$ standard deviation; $S B P$ systolic blood pressure; $D B P$ diastolic blood pressure; $B M I$ body mass index; $A C E 2$ angiotensinconverting enzyme 2; RFU relative fluorescence unit; $H T N$ hypertension; DM diabetes mellitus; $I H D$ ischemic heart disease; $C H F$ congestive heart failure; $P A D$ peripheral artery disease; $A C E i$ angiotensin-converting enzyme inhibitors; $A R B s$ angiotensin II receptor blocker; $\mathrm{Cr}$ creatinine 
Table 2 Multivariate linear regression models examining correlates of log (ACE2) serum levels in InCHIANTI participants aged 55 and older $(n=790)$

\begin{tabular}{|c|c|c|c|c|c|c|c|c|}
\hline & \multicolumn{2}{|c|}{ Model 1} & \multicolumn{2}{|c|}{ Model 2} & \multicolumn{2}{|c|}{ Model 3} & \multicolumn{2}{|c|}{ Model 4} \\
\hline & $\mathrm{SD} B$ & $p$ & $\mathrm{SD} B$ & $p$ & $\mathrm{SD} B$ & $p$ & $\mathrm{SD} B$ & $p$ \\
\hline Age & -0.12 & 0.0004 & -0.13 & 0.0003 & -0.19 & $<.0001$ & -0.16 & $<.0001$ \\
\hline Sex & 0.05 & 0.1504 & 0.06 & 0.1094 & -0.01 & 0.9191 & - & - \\
\hline HTN & & & 0.06 & 0.1407 & 0.074 & 0.0852 & 0.08 & 0.0665 \\
\hline $\mathrm{DM}$ & & & -0.03 & 0.3178 & -0.03 & 0.3265 & - & - \\
\hline PAD & & & 0.08 & 0.0222 & 0.09 & 0.0214 & 0.08 & 0.0241 \\
\hline ACEI & & & -0.13 & 0.0016 & -0.14 & 0.0013 & -0.14 & 0.0011 \\
\hline Weight & & & & & -0.06 & 0.1579 & -0.09 & 0.0186 \\
\hline Height & & & & & 0.02 & 0.7924 & - & - \\
\hline Smoking & & & & & -0.04 & 0.3116 & - & - \\
\hline Cr clearance & & & & & -0.06 & 0.1829 & - & - \\
\hline Total cholesterol & & & & & 0.01 & 0.6975 & - & - \\
\hline Comorbidity index & & & & & 0.04 & 0.2892 & - & - \\
\hline
\end{tabular}

HTN hypertension; $D M$ diabetes mellitus; $I H D$ ischemic heart disease; $C H F$ congestive heart failure; $P A D$ peripheral artery disease; $A C E i$ angiotensin-converting enzyme inhibitors; $A R B S$ angiotensin II receptor blocker; $\mathrm{Cr}$ creatinine

levels. These associations remained significant after adjustment for age, sex, smoking, height, weight, renal function, cholesterol levels, and non-cardiovascular comorbidity index. Model 4, which only includes independent variables that contributed to the model fit, using backward elimination method, showed the same association observed in the previous model. In addition, weight was inversely associated with ACE2, while hypertension became marginally associated with greater ACE2 levels (STB $=0.08, p=0.0665$ ).

To explore the effect of inflammation on ACE2 in the older group, we explored its association with interleukin-6 (IL6) and C-reactive protein (CRP) adjusting for the variables that had remained significantly associated with ACE2 in the previous model (Table 3). Log (CRP) was not significantly associated with ACE2. After adjusting for covariates, $\log$ (IL6) was positively and independently associated with ACE2 after adjusting for covariates. We tested the association of $\log$ (IL6) with ACE2 separately in PAD and non-PAD participants. There was a strong association between log (ACE2) and log (IL6) among those with PAD, but this term was only marginally significant in those free of PAD (Table 3).

Table 3 Multivariate linear regression models examining associations between ACE2 and related proteins and inflammatory markers in participants aged 55 and older

\begin{tabular}{|c|c|c|c|c|c|c|}
\hline & \multicolumn{2}{|c|}{ Total $(n=780)$} & \multicolumn{2}{|c|}{ With PAD $(n=75)$} & \multicolumn{2}{|c|}{ Without PAD $(n=705)$} \\
\hline & $\mathrm{SD} B$ & $p$ & $\mathrm{SD} B$ & $p$ & $\mathrm{SD} B$ & $p$ \\
\hline Age & -0.17 & $<.0001$ & -0.21 & 0.0797 & -0.16 & $<.0001$ \\
\hline PAD & 0.07 & 0.0413 & - & - & - & - \\
\hline Weight & -0.10 & 0.0052 & -0.29 & 0.0192 & -0.09 & 0.0257 \\
\hline $\mathrm{ACEi}$ & -0.09 & 0.0103 & -0.02 & 0.8336 & -0.09 & 0.0143 \\
\hline $\mathrm{ARB}$ & 0.09 & 0.0115 & 0.22 & 0.0518 & 0.08 & 0.0337 \\
\hline $\log ($ IL6) & 0.09 & 0.0186 & 0.25 & 0.0279 & 0.07 & 0.0726 \\
\hline
\end{tabular}

Initial models adjusted for CRP but were removed from the model for $p$ value $>0.1$

$A C E i$ angiotensin-converting enzyme inhibitors; $A R B$ angiotensin receptor blocker 


\section{Discussion}

\section{Principal findings}

This is the first study in humans to show a curvilinear association between circulating ACE2 level and age, with lower ACE2 level among older individuals. ACEi was associated with lower circulating ACE2 levels. Weight was inversely correlated with ACE2. While there were no associations of ACE2 levels in diabetes status, participants with PAD were more likely to have elevated ACE2 levels. Hypertensive individuals demonstrated a trend toward slightly higher circulating ACE2 levels, but the association was only marginally significant. Interestingly, ACE2 was positively associated with IL-6, a marker of chronic inflammation, but only in those with PAD.

Age differences in circulating ACE2 levels

ACE2 showed a curvilinear association with age characterized by a positive association among participants below the age of 55 and a negative association in older participants. This finding further expands the limited data on age-associated changes in ACE2. It is important to recognize the difficulty in interpreting changes in circulating ACE2 given the complex processes that determine it, the variable RAAS system it could represent in young and old. As a predominantly epithelial cell membrane protein, circulating ACE2 is mainly a product of the shedding of its surface domain cleaved by ADAM-17 [16]. Hence, the age-associated differences in circulating ACE2 levels we have observed represent the sum of age-associated differences in both the abundance of tissue cell membrane ACE2 and ADAM-17mediated shedding rate. Tissue levels of ACE2 were not measured in this study; thus, we cannot differentiate between the two processes using the available data.. While further studies are needed to better understand the implications of these age-associated differences, there are existing data that support the findings from the current study and shed light into the mechanism underlying the lower ACE2 in older age observed. Animal studies have shown lower ACE2 levels in the lungs of older compared with younger rats [7]. Similar inverse associations between ACE2/ACE levels and age were observed in hematopoietic stem progenitor cells in healthy adults [17]. It has been shown that ADAM-17mediated shedding of ACE2 is mainly driven by angiotensin $\mathrm{II}^{16}$. Given the overwhelming evidence of angiotensin II upregulation with aging [18], it would be expected that there is increased shedding resulting in greater circulating ACE2 with aging; however, this was not observed in this study. This suggests that lower circulating ACE2 at advanced age observed represents a decline in tissue ACE2 rather than a decline in shedding.

The age-associated differences observed in ACE2 are in line with established pro-inflammatory changes in renin-angiotensin signaling with aging, which leads to increased angiotensin II signaling with advancing age $[19,20]$. However, the lack of measurement of other proteins related to ACE2, namely angiotensin 1-7 and others downstream of angiotensin 1-7, prevents the full understanding of the biological implications of ACE2 associations observed here. Nonetheless, this ageassociated reduction in ACE2 is likely associated with an overall reduction in its ant-inflammatory effects with a net increase in pro-inflammatory signaling.

The emerging epidemiological data $[1,3,21]$ showed an overall curvilinear association between age and COVID-19 incidence. While a causal relationship between the complex pattern of ACE2 and age with COVID-19 incidence and severity cannot be claimed, it is noted that similar curvilinear patterns are observed. There are no differences in average ACE2 levels between the age group studies which would not support the role of ACE2 in mediating the differences in COVID-19 incidence and severity. However, given the complexity of the processes that would determine ACE2 circulating levels discussed above, the patterns of age differences in ACE2 within the age groups, rather than group averages, might better inform about the role of ACE2 in the disease pathogenesis.

With a plethora of social and environmental factors at play, ACE2 level is certainly not the only factor associated with the incidence pattern; however, given the major role of ACE2 as the protein that mediates viral entry into cells, it is plausible that the trends in ACE2 inform about a degree of biological predisposition. It is important to note, however, that circulating ACE2 levels are likely to represent different pro-inflammatory states in older vs. younger persons. Hence, circulating ACE2 levels tell only a part of the story in terms of predisposition to greater COVID-19 severity in older vs. younger individuals. Comprehensive assessment of RAAS signaling and the associated pro-inflammatory profiles in older and younger individuals will be needed to fully elucidate this complex relationship. 
The associations between ACE2 levels and the use of renin-angiotensin-aldosterone antagonists

This analysis showed that angiotensin-converting enzyme inhibitor (ACEi) use was associated with lower circulating ACE2 in this cohort; the small number of individuals on ARBs and aldosterone blockers in this cohort prevented deriving statistically reliable estimates for their association with ACE2 levels. While the molecular mechanism of the association of ACEi and circulating ACE2 levels cannot be explored in the current study, these findings are consistent with ACEi mechanism of actions. Knowledge on the feedback mechanisms between angiotensin II and ACE2 is still limited; however, there is evidence that angiotensin II increases the expression of ACE2 [22] as well as its shedding [16]; hence, low angiotensin II levels with ACEi are expected to reduce and downregulate these processes, leading to less circulating ACE2.

Initial concerns about the safety of RAAS antagonists were based on previous reports of elevated ACE2 in animals treated with ARBs [23], and the fear of subsequently increased virus virulence [23]. More extensive reviews of the literature, however, have shown mixed results on the changes in ACE2 with ACEi/ARB treatment [11]. Furthermore, evidence from animal models suggested beneficial effects of RAAS antagonists in reducing acute lung injury in coronavirus diseases [5, 11]. New observational evidence from this outbreak showed no adverse outcomes of RAAS antagonists $[24,25]$, and some suggesting beneficial role of these medications [26]. Several ongoing clinical trials are examining the efficacy of RAAS antagonists in preventing COVID-19 and/or reducing its severity.

ACE2 levels, weight, cardiovascular diseases, and inflammation in older individuals

Among the different cardiovascular risk factors examined in this analysis, weight was the only correlate of ACE2 with greater weight associated with lower levels in the older group. Interestingly, no associations were observed by BMI groups (data not shown). Preliminary epidemiological reports [27, 28] and anecdotal observations [29] have shown a link between obesity and severe COVID-19 severity. Further studies are needed to further examine the nature of the association of weight and ACE2 levels, and whether the suggested worse COVID-
19 outcomes among obese patients is mediated in part by lower ACE2 levels in this population.

The protective role of ACE2 in cardiovascular disease has been well recognized [30, 31]. However, greater circulating ACE levels have been reported in other chronic, pro-inflammatory $\mathrm{CV}$ conditions such as hypertension [32, 33], coronary artery disease [34], CHF $[33,35]$, and CKD [36]. In our sample, the association of ACE2 and cardiovascular disease was not tested due to low prevalence of ischemic heart disease and CHF. Further, there were no associations of ACE2 with hypertension or diabetes. However, PAD was associated with higher ACE2 levels in the older group. This association appears to be linked to chronic inflammation, with an association between ACE2 and IL-6 that was more significant in those with compared with those without PAD. The greater circulating ACE2 levels observed in individuals with disease can be a response by the marked pro-inflammatory, and angiotensin II upregulation [37], which has been associated with a compensatory increased ACE2 expression [22] and shedding in the circulation [16]. Further study of these associations in larger cohorts with higher disease prevalence is needed to confirm the associations of ACE2 with chronic conditions. Understanding the relationship between disease and ACE2 is essential in disentangling the changes in circulating ACE2 levels observed in COVID-19.

\section{Limitations}

There are limitations that one must consider while interpreting the results of this analysis. First, it is a cross-sectional study; hence, associations observed are amenable to selection bias. Second, the results of the study should be interpreted with caution given the assay utilized does not provide absolute quantification of ACE2, which might have limited the quality of the data; more studies using fully quantitative methods with different assays to assess ACE2 levels are needed to validate results from the proteomics approach and confirm our findings. In addition, further studies that measure other proteins in the angiotensin-renin cascade could provide further insight into the mechanisms of ACE2 with age. Third, the number of participants on ARBs was small; hence, the results, while statistically significant, could be a result of chance. Fourth, the complexity of RASS feedback signaling, however, and the tissuespecific differences make it imperative to employ caution while interpreting the differences observed in 
circulating ACE2 level in the absence of measures of other components of the RAAS systems.

\section{Conclusion}

This is the first study in humans to age-associated differences in ACE2 in a community-dwelling population, with lower ACE2 levels in older individuals. ACEi use was associated with lower ACE2. While weight was associated with lower circulating ACE2 levels, peripheral arterial disease was associated with greater circulating ACE2 levels, with a trend toward greater circulating ACE2 levels among hypertensives. There was a stronger positive association between ACE2 and chronic inflammation in those with PAD. Further studies are needed to examine the implications of these ageassociated differences in COVID-19 in cohorts with ACE2 levels and COVID-19 outcomes. More detailed characterization of the different components of RAS signaling is needed with a focus on absolute quantitative assessment in larger cohort toward establishing normative values, and assessment of their longitudinal changes.

Funding This research was supported entirely by the Intramural Research Program of the NIH, National Institute on Aging.

\section{References}

1. Bialek S, et al. Severe outcomes among patients with coronavirus disease 2019 (COVID-19) — United States, February 12-March 16, 2020. MMWR Morb Mortal Wkly Rep. 2020;69:343-6.

2. $\mathrm{Xu} \mathrm{X}-\mathrm{W}$, et al. Clinical findings in a group of patients infected with the 2019 novel coronavirus (SARS-Cov-2) outside of Wuhan, China: retrospective case series. BMJ. 2020;368:m606.

3. Boehmer TK, DeVies J, Caruso E, van Santen KL, Tang S, Black CL, et al. Changing age distribution of the COVID-19 pandemic-United States, May-August 2020. MMWR Morb Mortal Wkly Rep. 2020;69:1404-9.

4. Tokars JI, Olsen SJ, Reed C. Seasonal incidence of symptomatic influenza in the United States. Clin Infect Dis. 2018;66:1511-8.

5. AlGhatrif M, Cingolani O, Lakatta EG. The dilemma of coronavirus disease 2019, aging, and cardiovascular disease insights from cardiovascular aging science. JAMA Cardiol. 2020;5:747-8.

6. Zheng Y-Y, Ma Y-T, Zhang J-Y, Xie X COVID-19 and the cardiovascular system. Nat Rev Cardiol 1-2 (2020) doi: https://doi.org/10.1038/s41569-020-0360-5
7. Xudong X, Junzhu C, Xingxiang W, Furong Z, Yanrong L. Age- and gender-related difference of ACE2 expression in rat lung. Life Sci. 2006;78:2166-71.

8. Liang W, Zhu Z, Guo J, Liu Z, He X, Zhou W, et al. Severe acute respiratory syndrome, Beijing, 2003. Emerg Infect Dis. 2004;10:25-31.

9. Bunyavanich S, Do A, Vicencio A. Nasal gene expression of angiotensin-converting enzyme 2 in children and adults. JAMA. 2020;323:2427-9.

10. Li Y, et al. Angiotensin-converting enzyme 2 prevents lipopolysaccharide-induced rat acute lung injury via suppressing the ERK1/2 and NF- $\mathrm{KB}$ signaling pathways. Sci Rep. 2016;6:1-14.

11. Vaduganathan M, Vardeny O, Michel T, McMurray JJV, Pfeffer MA, Solomon SD. Renin-angiotensin-aldosterone system inhibitors in patients with Covid-19. N Engl J Med. 2020;382:1653-9.

12. Candia J, Cheung F, Kotliarov Y, Fantoni G, Sellers B, Griesman T, et al. Assessment of variability in the SOMAscan assay. Sci Rep. 2017;7:14248.

13. Tanaka T, Biancotto A, Moaddel R, Moore AZ, GonzalezFreire M, Aon MA, et al. Plasma proteomic signature of age in healthy humans. Aging Cell. 2018;17:e12799.

14. Aboyans V, Criqui MH, Abraham P, Allison MA, Creager MA, Diehm C, et al. Measurement and interpretation of the ankle-brachial index: a scientific statement from the American Heart Association. Circulation. 2012;126:2890 909.

15. Fabbri E, Zoli M, Gonzalez-Freire M, Salive ME, Studenski SA, Ferrucci L. Aging and multimorbidity: new tasks, priorities, and frontiers for integrated gerontological and clinical research. J Am Med Dir Assoc. 2015;16:640-7.

16. Patel VB, Clarke N, Wang Z, Fan D, Parajuli N, Basu R, et al. Angiotensin II induced proteolytic cleavage of myocardial ACE2 is mediated by TACE/ADAM-17: a positive feedback mechanism in the RAS. J Mol Cell Cardiol. 2014;66:167-76.

17. Aging healthy, or with diabetes, is associated with ACE2/ ACE imbalance in the hematopoietic stem progenitor cells | The FASEB Journal. https://www.fasebj. org/doi/abs/10.1096/fasebj.2019.33.1_supplement.514.7.

18. Wang M, Jiang L, Monticone RE, Lakatta EG. Proinflammation: the key to arterial aging. Trends Endocrinol Metab. 2014;25:72-9.

19. Lakatta EG. The reality of getting old. Nat Rev Cardiol. 2018;15:499-500.

20. Lakatta EG, Levy D. Arterial and cardiac aging: major shareholders in cardiovascular disease enterprises. Circulation. 2003;107:346-54.

21. Coronavirus: why it's so deadly in Italy - Andreas Backhaus Medium. https://medium.com/@andreasbackhausab/coronaviruswhy-its-so-deadly-in-italy-c4200a15a7bf.

22. Lin CS, Pan CH, Wen CH, Yang TH, Kuan TC. Regulation of angiotensin converting enzyme II by angiotensin peptides in human cardiofibroblasts. Peptides. 2010;31:1334-40.

23. Fang L, Karakiulakis G, Roth M. Are patients with hypertension and diabetes mellitus at increased risk for COVID-19 infection? Lancet Respir Med. 2020;8:e21.

24. Mehra MR, Desai SS, Kuy S, Henry TD, Patel AN. Cardiovascular disease, drug therapy, and mortality in Covid-19. N Engl J Med. 2020;382:e102. 
25. Fosbøl EL, Butt JH, Østergaard L, Andersson C, Selmer C, Kragholm K, et al. Association of angiotensin-converting enzyme inhibitor or angiotensin receptor blocker use with COVID-19 diagnosis and mortality. JAMA - J Am Med Assoc. 2020;324:168-77.

26. Meng J, Xiao G, Zhang J, He X, Ou M, Bi J, et al. Reninangiotensin system inhibitors improve the clinical outcomes of COVID-19 patients with hypertension. Emerg Microbes Infect. 2020;9:757-60.

27. Chow N, et al. Preliminary estimates of the prevalence of selected underlying health conditions among patients with coronavirus disease 2019-United States, February 12March 28, 2020. MMWR Morb Mortal Wkly Rep. 2020;69:382-6.

28. Kass DA, Duggal P, Cingolani O. Obesity could shift severe COVID-19 disease to younger ages. Lancet. 2020;395: 1544-5.

29. Obesity linked to severe coronavirus disease, especially for younger patients - The New York Times. https://www. nytimes.com/2020/04/16/health/coronavirus-obesityhigher-risk.html.

30. Raizada MK, Ferreira AJ. ACE2: a new target for cardiovascular disease therapeutics. J Cardiovasc Pharmacol. 2007;50:112-9.

31. Hamming I, Cooper ME, Haagmans BL, Hooper NM, Korstanje R, Osterhaus ADME, et al. The emerging role of ACE2 in physiology and disease. J Pathol. 2007;212:1-11.

32. Úri K, Fagyas M, Mányiné Siket I, Kertész A, Csanádi Z, Sándorfi G, et al. New perspectives in the renin-angiotensin- aldosterone system (RAAS) IV: circulating ACE2 as a biomarker of systolic dysfunction in human hypertension and heart failure. PLoS One. 2014;9:e87845.

33. Úri $\mathrm{K}$ et al. Circulating ACE2 activity correlates with cardiovascular disease development. JRAAS - J ReninAngiotensin-Aldosterone Syst 17, (2016).

34. Ramchand J, Patel SK, Srivastava PM, Farouque O, Burrell LM Elevated plasma angiotensin converting enzyme 2 activity is an independent predictor of major adverse cardiac events in patients with obstructive coronary artery disease. (2018) doi:https://doi.org/10.1371/journal.pone.0198144.

35. Epelman S, Shrestha K, Troughton RW, Francis GS, Sen S, Klein AL, et al. Soluble angiotensin-converting enzyme 2 in human heart failure: relation with myocardial function and clinical outcomes. J Card Fail. 2009;15:565-71.

36. Anguiano L, Riera M, Pascual J, Valdivielso JM, Barrios C, Betriu A, et al. Circulating angiotensin-converting enzyme 2 activity in patients with chronic kidney disease without previous history of cardiovascular disease. Nephrol Dial Transplant. 2015;30:1176-85.

37. Wang M, Jiang L, Monticone RE, Lakatta EG Proinflammation: the key to arterial aging Chronic inflammation and arterial aging doi:https://oi.org/10.1016/j. tem.2013.10.002, 2014.

Publisher's note Springer Nature remains neutral with regard to jurisdictional claims in published maps and institutional affiliations. 